\title{
Recurrent furunculosis - challenges and management: a review
}

\section{Kristina Sophie Ibler Charles B Kromann}

Department of Dermatology, Roskilde Hospital, Copenhagen University, Denmark
Correspondence: Kristina Sophie Ibler Department of Dermatology, Roskilde Hospital, Copenhagen University,

Køgevej 7-13, 4000 Roskilde, Denmark Tel +45 4732 2600

Email ksi@regionsjaelland.dk
This article was published in the following Dove Press journal:

Clinical, Cosmetic and Investigational Dermatology

18 February 2014

Number of times this article has been viewed
Abstract: Furunculosis is a deep infection of the hair follicle leading to abscess formation with accumulation of pus and necrotic tissue. Furuncles appear as red, swollen, and tender nodules on hair-bearing parts of the body, and the most common infectious agent is Staphylococcus aureus, but other bacteria may also be causative. In some countries, methicillin resistant $S$. aureus is the most common pathogen in skin and soft tissue infections which is problematic since treatment is difficult. Furunculosis often tends to be recurrent and may spread among family members. Some patients are carriers of $S$. aureus and eradication should be considered in recurrent cases. Solitary lesions should be incised when fluctuant, whereas patients with multiple lesions or signs of systemic disease or immunosuppression should be treated with relevant antibiotics. The diagnostic and therapeutic approach to a patient suspected of staphylococcosis should include a thorough medical history, clinical examination, and specific microbiological and biochemical investigations. This is particularly important in recurrent cases where culture swabs from the patient, family members, and close contacts are mandatory to identify and ultimately control the chain of infection. Focus on personal, interpersonal, and environmental hygiene issues is crucial to reduce the risk of contamination and recurrences.

Keywords: furunculosis, MRSA, SSTI, boils, abscess, staphylococcosis

\section{Introduction}

Furunculosis is a deep infection of the hair follicle leading to abscess formation with accumulation of pus and necrotic tissue. Furuncles appear on the hair-bearing parts of the skin and the infectious agent is Staphylococcus aureus. However, other bacteria may also be causative. ${ }^{1}$ Furunculosis occurs independently of methicillin resistant S. aureus (MRSA) infection, which has become endemic in some countries. MRSA are more difficult to treat with standard antibiotics and hence pose a specific clinical and microbiological problem, which is dealt with in great detail elsewhere and will therefore not be described in detail here. ${ }^{2}$

\section{Signs and symptoms}

Clinically, furuncles present as red, swollen, and tender nodules of varying size and at times with an overlying pustule. Fever and enlarged lymph nodes are rare. If several adjacent follicles are infected they may coalesce and form a larger nodule, known as a carbuncle. Furuncles most frequently appear on the extremities and they may lead to scarring upon healing. ${ }^{3}$ Most patients present with one or two boils and after clearing experience no recurrences. However, furunculosis has a propensity to recur and in such cases they often spread among family members. 


\section{Recurring furunculosis}

Recurrent furunculosis is generally defined as three or more attacks within a 12 month period. ${ }^{4}$ Colonization of $S$. aureus in the anterior nares plays a definite role in the etiology of chronic or recurrent furunculosis. Besides the nares, colonization also occurs in warm, moist skin folds such as behind ears, under pendulous breasts, and in the groin. Bacteria other than $S$. aureus may also be pathogenic, especially for furuncles in the vulvovaginal and perirectal area, and on the buttocks. ${ }^{1}$ Especially, enteric species such as Enterobacteriaceae and Enterococci are often present at these sites. Corynebacterium, S. epidermidis, and S. pyogenes may also be present in furunculosis. Immunodeficiency is rarely the primary cause.

Quality of life in patients with recurrent furunculosis has, to our knowledge, not been investigated. However, decreased quality of life was found in MRSA positive patients who were isolated in palliative institutions ${ }^{5}$ and in patients with other recurrent boil diseases like hidradenitis suppurativa. ${ }^{6,7}$

\section{Risk factors}

Direct physical contact with infected individuals, primarily family members or health care personnel, is the main risk factor for development of furunculosis. Risk factors associated with recurrent furunculosis were investigated in a case control study including 74 patients with recurrent furunculosis and an equal number of patients with nonrecurrent furunculosis. ${ }^{4}$ Nasal swabs revealed S. aureus in $89 \%$ and $100 \%$ of recurrent and nonrecurrent furunculosis, respectively, and no significant differences were detected in resistance to the commonly used antibiotics. The most important independent predictor of recurrence was a positive family history. Other independent predictors were anemia, previous antibiotic therapy, diabetes mellitus, previous hospitalization, multiplicity of lesions, poor personal hygiene, and associated diseases. ${ }^{4}$ Established skin diseases such as atopic dermatitis, chronic wounds, or leg ulcers increase the susceptibility to bacterial colonization and are more prone to develop furunculosis. ${ }^{8}$ Deficiency of mannose-binding lectin as well as impaired neutrophil function in mentally retarded adults have also been associated with furunculosis. ${ }^{9-11}$ Obesity and hematological disorders are also predisposing factors. Nonetheless, in the majority of cases, no convincing predisposing factors can be incriminated.

\section{MRSA}

Recurrent furunculosis is most often caused by methicillin susceptible $S$. aureus. However, community acquired MRSA
(CA-MRSA) has become endemic in the US and is now the most common cause of soft tissue infection in the emergency room in many countries. ${ }^{12-15}$ The prevalence of CA-MRSA is higher in the US when compared to Europe but the prevalence in Europe is rising. ${ }^{16-21}$

Some strains of MRSA, particularly the CA-MRSA, produce a toxin named Panton-Valentine leukocidin (PVL) and are associated with severe infections. ${ }^{22} \mathrm{PVL}$ is leucocidal, and severe but rare complications such as necrotizing fasciitis and necrotizing pneumonia are described following soft tissue infection with MRSA. PVL is a virulence factor of $S$. aureus which correlates with chronic recurrent furunculosis. ${ }^{23-26}$

\section{Diagnosis}

The diagnosis of furunculosis is relatively straight forward. The microbial agent can be identified with simple cultured swabs. A general clinical examination should be performed, and investigations not only involve culture swabs of the lesions (preferably from pus or fluids from fluctuant boils, eventually obtained by incision) but also of the carrier sites such as nostrils and perineum. Depending on the history, culture swabs of the family members may be relevant. It is suggested to investigate urine and blood glucose, or glycated hemoglobin $\left(\mathrm{HbA}_{1 \mathrm{c}}\right)$ to identify any underlying diabetes, and a full blood count to exclude systemic infection or other internal disease. Immunological evaluation may be considered in recurrent disease or signs of internal disease.

\section{Differential diagnosis}

If the nodules are exclusively located in the axillae, the groin, and/or in inframammary regions, hidradenitis suppurativa (HS) should be considered as a differential diagnosis. History taking of personal as well as familial episodes of boils is important. In women, intensified symptoms associated with monthly periods is a telltale sign of HS, and HS may over time, lead to sinus tracts and fistulas with malodorous putrid discharge. Other differential diagnoses include foreign body reactions, pilonidal cysts, abscesses of Bartholin's glands, and other kinds of abscesses.

\section{Complications}

The most common complications to furunculosis are scarring and recurrence. Rarely does furunculosis lead to systemic infection with fever and organ related symptoms. Positive blood culture and endocarditis following furunculosis are described. ${ }^{27}$ Skin infection with MRSA has been shown to be complicated by systemic infection including respiratory distress and pneumonia, ${ }^{23}$ and necrotizing fasciitis and myositis 
are also reported. ${ }^{26}$ Osteomyelitis, septic arthritis, and central nervous system infections with meningitis and brain abscess following infection with $S$. aureus are also reported. ${ }^{28-33}$

\section{Treatment}

The management of recurrent furunculosis is problematic and may be disappointing. A flowchart on the management of furunculosis is shown in Figure 1. Simple incision and drainage may be sufficient in solitary lesions, but systemic antibiotic therapy may be required. $S$. aureus has the ability of developing resistance to different antibiotics, and this is important to bear in mind when choosing antimicrobials. ${ }^{34}$

According to clinical practice guidelines on infections with MRSA, incision and drainage alone is likely to be adequate for simple abscesses or boils, but additional research is needed to further define the role of antibiotics, if any, in this setting. ${ }^{35,36}$

Antibiotics are recommended if the skin infection is associated with severe disease (multiple sites of infection or rapid progression), systemic illness, or associated comorbidities or immunosuppression, extremes of age, abscess in an area difficult to drain (eg, face, hand, and genitalia), associated septic phlebitis, and lack of response to incision and drainage alone.

Guidelines by the Infectious Diseases Society of America suggest the following oral antibiotics for empirical coverage of CA-MRSA in outpatients: clindamycin, trimethoprim-sulfamethoxazole, a tetracycline (doxycycline or minocycline), and linezolid. ${ }^{35}$ If coverage for both $\beta$-hemolytic streptococci and CA-MRSA is desired, options include clindamycin alone, or trimethoprimsulfamethoxazole, or a tetracycline in combination with a $\beta$-lactam (eg, amoxicillin), or linezolid alone. ${ }^{35}$

For hospitalized patients with complicated infection, in addition to surgical debridement and broad spectrum antibiotics, empirical therapy for MRSA should be considered while awaiting culture data. Options include intravenous (IV) vancomycin $1 \mathrm{~g}$ twice daily, oral or IV linezolid $600 \mathrm{mg}$

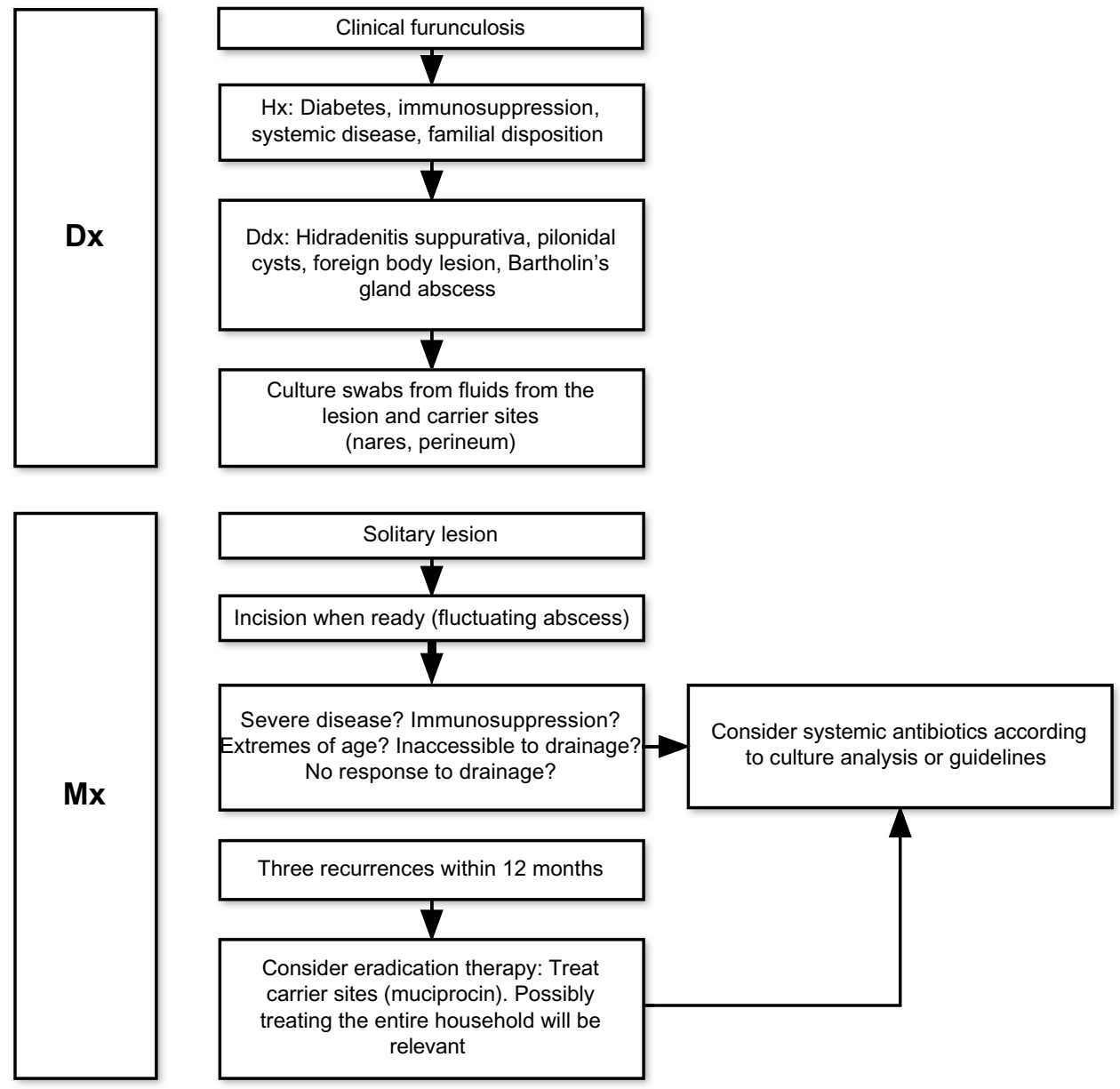

Figure I Flowchart of diagnosis and treatment of furunculosis.

Abbreviations: Ddx, differential diagnosis; Dx, diagnosis; Hx, history; Mx, management. 
twice daily, daptomycin $4 \mathrm{mg} / \mathrm{kg} /$ dose IV once daily, telavancin $10 \mathrm{mg} / \mathrm{kg} /$ dose IV once daily, and clindamycin $600 \mathrm{mg}$ IV or oral three times a day. A $\beta$-lactam antibiotic (eg, cefazolin) may be considered in hospitalized patients with nonpurulent cellulitis. Adjustment to MRSA-active therapy must be initiated if there is insufficient clinical response. ${ }^{35}$ Seven to 14 days of therapy is recommended but should be individualized on the basis of the patient's clinical response. Hospitalized patients with MRSA must be isolated from other patients.

\section{Prevention}

Preventive educational information on personal hygiene and appropriate wound care are recommended for patients with skin or soft tissue infections (SSTI) such as recurrent furunculosis. ${ }^{37,38}$ Wounds should be covered with clean, dry bandages and good personal hygiene with regular bathing and washing of hands with soap and water, or cleansing with an alcohol-based hand gel is recommended, particularly after touching infected skin or an item that has directly contacted a draining wound. Recycling or sharing personal items such as disposable or electrical razors and epilators, linens, and towels that have been used on infected skin should be avoided. Environmental hygiene measures should be considered in patients with recurrent SSTI in the household or community setting. Cleaning efforts should be focused on frequently touched surfaces (ie, counters, door knobs, bath tubs, and toilet seats) that may contact bare skin or uncovered infections. Commercially available cleaners or detergents appropriate for the surface being cleaned should be used according to product instructions for routine cleaning of surfaces. ${ }^{35}$

\section{Colonization}

Topical attempts at decolonizing with mupirocin and chlorhexidine can reduce the incidence of subsequent $S$. aureus infections although with variable efficacy. ${ }^{39-42}$ Decolonization may be considered in cases with recurrent SSTI despite optimizing wound care and hygiene measures, and in cases with ongoing transmission among household members or other close contacts despite hygiene measures. Decolonization usually consists of 5 to 10 days application of mupirocin ointment twice daily in the nostrils and daily body wash with $4 \%$ chlorhexidine soap for 5 to 14 days. Dilute bleach baths 15 minutes twice daily for 3 months can be considered. Oral rinsing with $0.2 \%$ chlorhexidine solution three times daily decreases pharyngeal flora. ${ }^{41}$ Topical gentian violet $0.3 \%$ solution to the nostrils twice daily for 2 to 3 weeks has also been suggested. ${ }^{40}$
Oral antimicrobial therapy is recommended for the treatment of active infection only and is not routinely recommended for decolonization. An oral agent in combination with rifampicin, if the strain is susceptible, may be considered for decolonization if infections recur despite the above mentioned topical measures. ${ }^{35}$ Rifampicin monotherapy is at risk of selecting resistant variants and is not recommended. The combination of topical and systemic antimicrobials is highly effective with a clearance rate of $87 \%$ in treated patients. ${ }^{43}$ Rectal swab cultures may be considered in refractory cases since the gastrointestinal tract may be a reservoir of methicillin susceptible $S$. aureus and MRSA. In these cases, oral vancomycin (1 g twice daily for 5 days) can eradicate $80 \%-100 \%$ of MRSA gut colonization. ${ }^{43}$ Urogenital and vaginal colonization may also appear.

If family members are carriers they should be treated as the patient. Besides decolonization, any impairment of the patients' nutritional status should be improved if at all possible.

\section{Discussion and conclusion}

In healthy individuals the human microbiome differs remarkably in the skin and other habitats. This diversity is not fully understood, but host genetics, the environment, and early microbial exposure are implicated. Thus, many carriers of nasal staphylococci do not develop furuncles or boils whereas others do. Furunculosis is a skin condition which tends to be recurrent and often spreads to family members either directly by skin contact or indirectly. The infection is most often caused by $S$. aureus and resistance toward antimicrobials is an increasing problem. MRSA is now endemic in many countries, and constitutes an emerging problem worldwide.

The morbidity associated with furunculosis may be considerable. Treatment is therefore important. Clinically, serious complications to SSTIs with $S$. aureus include bacteremia, infective endocarditis, and necrotizing pneumonia. Skin complications include purulent ulceration and pain from lesions, which is traditionally perceived as stigmatizing since the descriptions in the Book of Job (The Hebrew Bible). To the best of the authors' knowledge, there are no quality of life studies in patients with recurrent furunculosis. In a recent German survey of patients in palliative care units isolated due to MRSA, a negative impact on quality of life was found, and the necessary isolation was considered to be a significant burden at the end of life in these terminal patients. ${ }^{5}$ Most patients are, however, not 
isolated due to their skin infections, and other aspects may therefore play a role. It may be speculated that clinical similarities between furunculosis, HS, and acne vulgaris, may lead to negative influences on quality of life. Several studies have identified both acne and HS as major sources of quality of life reduction in patients, suggesting that a similar relationship may exist for furunculosis. In consequence, it is suggested that specific quality of life studies are needed for furunculosis as well.

The diagnostic and therapeutic approach to a patient suspected of staphylococcosis should include a thorough medical history, clinical examination, and specific microbiological and biochemical investigations. This is particularly important if recurrent lesions are a problem. In such cases it is mandatory to collect culture swabs from the patient, family members, and close contacts to identify and ultimately control the chain of infection. Focus on personal, interpersonal, and environmental hygiene issues is crucial to reduce the risk of contamination and recurrences. Specific attention should be paid to improving and maintaining the skin barrier. An intact skin barrier function reduces the risk of infection and regular use of emollients to keep the skin moist is therefore helpful.

Several therapeutic modalities are relevant to the management of staphylococcosis. Traditionally, incision and drainage has been used extensively. This procedure should however be restricted to fluctuant boils. Nonfluctuant boils generally do not benefit from incision and drainage. Once incised, the abscess cavity may require packing to maintain drainage. Minor elements that do not fluctuate do not need incision and can be managed by keeping the skin area clean and protected.

If fever is present or if the patient develops systemic signs of infection, systemic antimicrobials are needed. Regular and frequent swabbing cultures of the lesions are helpful in deciding which antimicrobial to choose as the microbial resistance pattern may vary according to time, location, and geography.

Ultimately, treatment of staphylococcosis depends on eradication of pathogenic strains in patients and carriers. Eradication should, however, be restricted to patients or families with recurrent SSTI. In most cases, colonization with $S$. aureus is not harmful, and the high number of asymptomatic carriers contradicts eradication in this population. Further studies are needed to elucidate the microbiome complexity in carriers of $S$. aureus, and to elucidate the effect and mechanisms of using, eg, probiotics, rather than antibiotics for bacterial population control.

\section{Disclosure}

The authors report no conflicts of interest in this work.

\section{References}

1. Dahl MV. Strategies for the management of recurrent furunculosis. South Med J. 1987;80:352-356.

2. Pottinger PS. Methicillin-resistant Staphylococcus aureus infections. Med Clin North Am. 2013;97:601-619.

3. Taira BR, Singer AJ, Thode HC Jr, Lee CC. National epidemiology of cutaneous abscesses: 1996 to 2005. Am J Emerg Med. 2009;27:289-292.

4. El-Gilany AH, Fathy H. Risk factors of recurrent furunculosis. Dermatol Online J. 2009;15:16.

5. Bukki J, Klein J, But L, et al. Methicillin-resistant Staphylococcus aureus (MRSA) management in palliative care units and hospices in Germany: a nationwide survey on patient isolation policies and quality of life. Palliat Med. 2013;27:84-90.

6. Wolkenstein P, Loundou A, Barrau K, Auquier P, Revuz; Qualtiy of Life Group of the French Society of Dermatology. Quality of life impairment in hidradenitis suppurativa: a study of 61 cases. JAm Acad Dermatol. 2007;56:621-623.

7. Onderdijk AJ, van der Zee HH, Esmann S, et al. Depression in patients with hidradenitis suppurativa. J Eur Acad Dermatol Venereol. 2013;27: 473-478.

8. Hoeger PH. Antimicrobial susceptibility of skin-colonizing S. aureus strains in children with atopic dermatitis. Pediatr Allergy Immunol. 2004; 15:474-477.

9. Demircay Z, Eksioglu-Demiralp E, Ergun T, Akoglu T. Phagocytosis and oxidative burst by neutrophils in patients with recurrent furunculosis Br J Dermatol. 1998;138:1036-1038.

10. Gilad J, Borer A, Smolyakov R, Reisenberg K, Schlaeffer F, Levy R. Impaired neutrophil functions in the pathogenesis of an outbreak of recurrent furunculosis caused by methicillin-resistant Staphylococcus aureus among mentally retarded adults. Microbes Infect. 2006;8: $1801-1805$.

11. Kars M, van DH, Salimans MM, Bartelink AK, van de Wiel A. Association of furunculosis and familial deficiency of mannose-binding lectin. Eur J Clin Invest. 2005;35:531-534.

12. Moran GJ, Krishnadasan A, Gorwitz RJ, et al; EMERGEncy ID Net Study Group. Methicillin-resistant S. aureus infections among patients in the emergency department. N Engl J Med. 2006;355:666-674.

13. Moellering RC Jr. The growing menace of community-acquired methicillin-resistant Staphylococcus aureus. Ann Intern Med. 2006;144: 368-370.

14. Moellering RC Jr. Current treatment options for community-acquired methicillin-resistant Staphylococcus aureus infection. Clin Infect Dis. 2008;46:1032-1037.

15. Zetola N, Francis JS, Nuermberger EL, Bishai WR. Communityacquired meticillin-resistant Staphylococcus aureus: an emerging threat. Lancet Infect Dis. 2005;5:275-286.

16. Moxnes JF, de Blasio BF, Leegaard TM, Moen AE. Methicillin-resistant Staphylococcus aureus (MRSA) is increasing in Norway: a time series analysis of reported MRSA and methicillin-sensitive S. aureus cases, 1997-2010. PLoS One. 2013;8:e70499.

17. Faria NA, Oliveira DC, Westh H, et al. Epidemiology of emerging methicillin-resistant Staphylococcus aureus (MRSA) in Denmark a nationwide study in a country with low prevalence of MRSA infection. J Clin Microbiol. 2005;43:1836-1842.

18. Fossum AE, Bukholm G. Increased incidence of methicillin-resistant Staphylococcus aureus ST80, novel ST125 and SCCmecIV in the south-eastern part of Norway during a 12-year period. Clin Microbiol Infect. 2006;12:627-633.

19. Larsen AR, Stegger M, Bocher S, Sorum M, Monnet DL, Skov RL. Emergence and characterization of community-associated methicillinresistant Staphyloccocus aureus infections in Denmark, 1999 to 2006. J Clin Microbiol. 2009;47:73-78. 
20. Robinson DA, Kearns AM, Holmes A, et al. Re-emergence of early pandemic Staphylococcus aureus as a community-acquired meticillinresistant clone. Lancet. 2005;365:1256-1258.

21. Wannet WJ, Spalburg E, Heck ME, et al. Emergence of virulent methicillin-resistant Staphylococcus aureus strains carrying PantonValentine leucocidin genes in The Netherlands. J Clin Microbiol. 2005;43:3341-3345.

22. Frazee BW, Lynn J, Charlebois ED, Lambert L, Lowery D, Perdreau-Remington F. High prevalence of methicillin-resistant Staphylococcus aureus in emergency department skin and soft tissue infections. Ann Emerg Med. 2005;45:311-320.

23. Al-Tawfiq JA, Aldaabil RA. Community-acquired MRSA bacteremic necrotizing pneumonia in a patient with scrotal ulceration. $J$ Infect. 2005;51:e241-e243.

24. Gillet Y, Etienne J, Lina G, Vandenesch F. Association of necrotizing pneumonia with Panton-Valentine leukocidin-producing Staphylococcus aureus, regardless of methicillin resistance. Clin Infect Dis. 2008;47:985-986.

25. Lina G, Piemont Y, Godail-Gamot F, et al. Involvement of Panton-Valentine leukocidin-producing Staphylococcus aureus in primary skin infections and pneumonia. Clin Infect Dis. 1999;29: 1128-1132.

26. Miller LG, Perdreau-Remington F, Rieg G, et al. Necrotizing fasciitis caused by community-associated methicillin-resistant Staphylococcus aureus in Los Angeles. N Engl J Med. 2005;352:1445-1453.

27. Bahrain M, Vasiliades M, Wolff M, Younus F. Five cases of bacterial endocarditis after furunculosis and the ongoing saga of communityacquired methicillin-resistant Staphylococcus aureus infections. Scand J Infect Dis. 2006;38:702-707.

28. Chang WN, Lu CH, Wu JJ, et al. Staphylococcus aureus meningitis in adults: a clinical comparison of infections caused by methicillin-resistant and methicillin-sensitive strains. Infection. 2001;29:245-250.

29. Corrah TW, Enoch DA, Aliyu SH, Lever AM. Bacteraemia and subsequent vertebral osteomyelitis: a retrospective review of 125 patients. QJM. 2011;104:201-207.

30. Cosgrove SE, Sakoulas G, Perencevich EN, Schwaber MJ, Karchmer AW, Carmeli Y. Comparison of mortality associated with methicillin-resistant and methicillin-susceptible Staphylococcus aureus bacteremia: a metaanalysis. Clin Infect Dis. 2003;36:53-59.

31. Ferreira JP, Abreu MA, Rodrigues P, Carvelho L, Correia JA. [Meticilin resistant Staphylococcus aureus and liver abscess: a retrospective analysis of 117 patients]. Acta Med Port. 2011;24 Suppl 2:399-406. Portugese.
32. Gelfand MS, Cleveland KO. Vancomycin therapy and the progression of methicillin-resistant Staphylococcus aureus vertebral osteomyelitis. South Med J. 2004;97:593-597.

33. Huang PY, Chen SF, Chang WN, et al. Spinal epidural abscess in adults caused by Staphylococcus aureus: clinical characteristics and prognostic factors. Clin Neurol Neurosurg. 2012;114:572-576.

34. Nagaraju U, Bhat G, Kuruvila M, Pai GS, Jayalakshmi, Babu RP. Methicillin-resistant Staphylococcus aureus in community-acquired pyoderma. Int J Dermatol. 2004;43:412-414.

35. Liu C, Bayer A, Cosgrove SE, et al; Infectious Diseases Society of America. Clinical practice guidelines by the infectious diseases society of america for the treatment of methicillin-resistant Staphylococcus aureus infections in adults and children. Clin Infect Dis. 2011;52: e18-e55.

36. Nathwani D, Morgan M, Masterton RG, et al; British Society for Antimicrobial Chemotherapy Working Party on Community-onset MRSA Infections. Guidelines for UK practice for the diagnosis and management of methicillin-resistant Staphylococcus aureus (MRSA) infections presenting in the community. J Antimicrob Chemother. 2008;61:976-994.

37. Laube S, Farrell AM. Bacterial skin infections in the elderly: diagnosis and treatment. Drugs Aging. 2002;19:331-342.

38. Turabelidze G, Lin M, Wolkoff B, Dodson D, Gladbach S, Zhu BP. Personal hygiene and methicillin-resistant Staphylococcus aureus infection. Emerg Infect Dis. 2006;12:422-427.

39. Harbarth S, Dharan S, Liassine N, Herrault P, Auckenthaler R, Pittet D. Randomized, placebo-controlled, double-blind trial to evaluate the efficacy of mupirocin for eradicating carriage of methicillin-resistant Staphylococcus aureus. Antimicrob Agents Chemother. 1999;43:1412-1416.

40. Okano M, Noguchi S, Tabata K, Matsumoto Y. Topical gentian violet for cutaneous infection and nasal carriage with MRSA. Int $J$ Dermatol. 2000;39:942-944.

41. Ringberg H, Cathrine PA, Walder M, Hugo Johansson PJ. The throat: an important site for MRSA colonization. Scand J Infect Dis. 2006;38:888-893.

42. Simor AE, Phillips E, McGeer A, et al. Randomized controlled trial of chlorhexidine gluconate for washing, intranasal mupirocin, and rifampin and doxycycline versus no treatment for the eradication of methicillin-resistant Staphylococcus aureus colonization. Clin Infect Dis. 2007;44:178-185.

43. Atanaskova N, Tomecki KJ. Innovative management of recurrent furunculosis. Dermatol Clin. 2010;28:479-487.
Clinical, Cosmetic and Investigational Dermatology

\section{Publish your work in this journal}

Clinical, Cosmetic and Investigational Dermatology is an international, peer-reviewed, open access, online journal that focuses on the latest clinical and experimental research in all aspects of skin disease and cosmetic interventions. All areas of dermatology will be covered; contributions will be welcomed from all clinicians and

\section{Dovepress}

basic science researchers globally. This journal is indexed on CAS The manuscript management system is completely online and includes a very quick and fair peer-review system, which is all easy to use. Visit http://www.dovepress.com/testimonials.php to read real quotes from published authors. 